\title{
Reduction of Garments Bottleneck Processing Time on the Sewing Line of the Garments Industries
}

\author{
Sazid Elahi ${ }^{1}$, Md Dulal Hosen ${ }^{2 *}$, Farhana Jannat ${ }^{3}$, Nasrin Jamine ${ }^{3}$ and Syed Mahir Ali $^{3}$ \\ ${ }^{1}$ Department of Apparel Engineering, Bangladesh University of Textiles, Dhaka, Bangladesh \\ ${ }^{2}$ Department of Textile Engineering, Mawlana Bhashani Science and Technology University, Bangladesh \\ ${ }^{3}$ Department of Apparel Merchandising \& Management, BGMEA University of Fashion and Technology, Bangladesh
}

*Corresponding author: Md Dulal Hosen, Department of Textile Engineering, Mawlana Bhashani Science and Technology University, Dhaka, Bangladesh.

\begin{abstract}
Operation management is one among the foremost powerful technique for deciding in production floor management and therefore the sewing stage is that the most vital and crucial stage in garment production floor, involves tons of operations having a special cycle times within the traditional sewing line of clothes, all of the workers aren't equally expert to try to all the method. As a result, the problematic zones arise within the assembly line, which is typically referred to as the bottleneck workstation or operation. This study deals with bottleneck detection in sewing line. A bottle neck may be a point of congestion during a production system that happens when workloads arrive too quickly for the assembly process to handle, denotes as, rock bottom output point in assembly line. Reducing bottle neck is additionally related with increasing productivity it's proven that with the assistance of various bottleneck elimination technique like line balancing, lean application technique, has been possible to spot non-value-added process and eliminate them from production process. During this paper, we've taken the assembly data of various apparel products like sport shirt and T-shirt. By applying lean technique, ensures maximum space utilization, inventory \& transportation reduction and thus reduces cycle time \& increases efficiency. The experimental results show significant improvement in productivity and line efficiency as compared to the prevailing system.
\end{abstract}

Keywords: Method Study; Lean; SMV; Improvement; Productivity

\section{Introduction}

Over the past 150 years, garment structures have changed from the manual fitting and assembly of individual hand sewn garments to mechanized, automated and sometimes robotized for batch production. Recently, to satisfy customer's demands through the assembly of excellence garments product at lowest possible cost are the foremost focuses in RMG industry [1,2]. Appropriate planning or controlling system can contribute to understand better performance during this area. The assembly process of garments industry is often grouped into three main stages cutting, sewing and finishing. The stitching stage is that the foremost vital and crucial stage among them [3]. Sewing stages involves plenty of operations having a unique cycle time to perform within the traditional sewing line of garments, all of the workers aren't equally expert to undertake to all or any the tactic [2]. As a result, the problematic zones arise within the mechanical system, which is usually remarked because the bottleneck workstation or operation [4]. Bottleneck is an operation having longer cycle time and where process goes slow within the stitching mechanical system. This process that have bottleneck problem, is that the main reason for reducing the efficiency of the assembly line. Line balancing is one quite technique for balancing the assembly line. At present, traditional production system possesses to urge replaced with assembly lines for greater product variability and shorter cycle time. The aim of this study is to unravel the bottleneck problem of sewing line during a garment manufacturing company [1]. The layout of the road was modified using the road balancing and time and examination technique. The world elimination of quotient on has greatly enhanced the opportunities for sourcing from Bangladesh.

Bangladesh supplies over US $\$ 28$ billion worth of textiles and apparel to the world markets. And exports are growing rapidly as more and more buyers around the turn to India as an alternative to China. In 2005, spurred by the worldwide elimination of quotas 
shipments to the EU soared by $30 \%$ and people to the USA shot up by $34 \%$. These increases are remarkable, as long as EU imports from all sources rose by only $8 \%$ while US import growth was just $6 \%$ [5]. Consumer spending is slowing down everywhere the planet. Retailers are trying to find real innovation from their suppliers. They want really new garments made from new fabrics and yarns. They want new services to offer their customers [6].

Competition within the late 1990's are going to be supported the capabilities and core competences of textile and clothing companies and on the building of long-term supply relationships [7]. There are many opportunities to be addressed. Textile and clothing machinery will still be improved butt the foremost interesting technologies for the 2000s are within the areas of fibers, fabrics, measurement, control and multimedia. We can say an apparel industry is an independent industry from the essential requirement of staple to final products, with huge value addition at every stage of processing [8]. Apparel industry is of the total country export. The largest foreign exchange earning sector contributing 15\% in this scenario, the Bangladeshi garment industries have witnessed substantial improvements in recent years. But the unnecessary capital investment isn't getting to solve the matter entirely; moderately this may end up the waste in end of the day [9]. The aim of this study is to unravel the bottleneck problem of stitching line during a garment manufacturing company. For reducing bottleneck in apparel sewing line, different elimination techniques have been used [10]. Such as work sharing method, line balancing method, implementation of lean. We reveal that how above-mentioned techniques can be integrated to show a best picture of non-valueadded activities present in the system and, hereby eliminating the problem that causes bottleneck.

\section{Materials and Methods}

\section{Materials}

In this Research was conducted in a selected garment located in Tongi, Gazipur, Bangladesh. Here quantitative data used for the analysis of this research. The collected data tabled the data firstly in accordance with traditional operation break down then according to work sharing method and then Lean line operational break down. Finally, the results are compared with the help of Time study, Bar chart, Histogram etc. In this research some very normal materials are used to carry out the data collection and analysis. Those materials are:-
1. Statistical data
2. Foreign Trade Source
3. Paper
4. Pencil
5. Calculator
6. Pen

\section{Scale and}

8. Stop watch etc.

\section{Methods}

The following Line Balancing Techniques are being used in this research:-

- Method Improvement

- $\quad$ Share Capacity

- $\quad$ Add Additional Manpower or Machine

- Improve Workstation Layout

- Better Operator

- Work for Extra Hours

- $\quad$ Use Time Saving Tricks

- $\quad$ Reduce Bottleneck by Work Sharing Method

At first, a stitching line of t-shirts was chosen for balancing and required information was gathered from the road like number of operator and helper, cycle time, target, and SMV. Then we took the time of every process needed for the completion of every worker. Then we found out the bottleneck area. At the numbers 6, 10, 12, and 17 operators, the capacity was above the previous operators, thus, add process was high there. Two operators were reduced from the operation hem sleeve and attached sleeve to body and the number 6 operation was shared with number 5. Moreover, number 17 was removed and shared with the number 18 operator. Therefore, the work process development of line balancing is done by this flow process:-

Operation breakdown

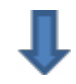

Leveling workload

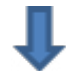

Calculating target, SMV, line efficiency

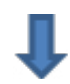

Find out bottleneck area and Relocate the workload

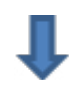

Calculate the line efficiency after balancing

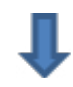

Represent the result graphically

\section{Results and Discussions}

\section{Experimental data (Before work-sharing)}

It is clear from the graph that in the 6,10,12, and 17 number operations, the bottleneck which occurred at those points' capacity was higher than the previous operation (Figure $1 \&$ Table 1). 


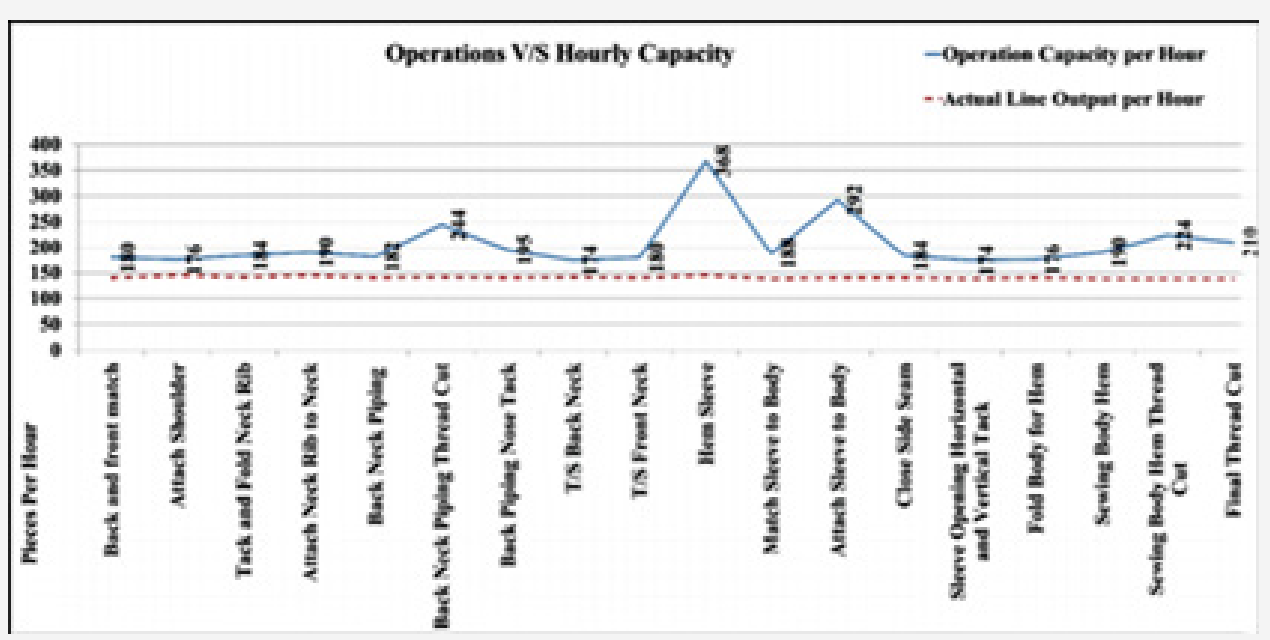

Figure 1: Operation vs. hourly capacity.

Table 1: Capacity study based pitched diagram.

\begin{tabular}{|c|c|c|c|c|c|c|c|c|c|}
\hline \multicolumn{10}{|c|}{ Mahadi Fashion (Pvt) Ltd } \\
\hline \multicolumn{10}{|c|}{ Capacity study based pitch diagram } \\
\hline \multicolumn{2}{|c|}{ Buyer: Sisal } & \multirow{2}{*}{$\begin{array}{l}\text { Sty \# } 6704 \\
\text { Operations }\end{array}$} & \multirow{2}{*}{$\begin{array}{c}\text { SMV } \\
\text { No of } \\
\text { operators }\end{array}$} & \multirow{2}{*}{$\begin{array}{c}5.5 \\
\text { Cycle } \\
\text { time with } \\
\text { allowance } \\
\text { (In minutes) }\end{array}$} & \multirow[b]{2}{*}{$\begin{array}{c}\text { Operation } \\
\text { capacity per } \\
\text { hour }\end{array}$} & \multirow[b]{2}{*}{$\begin{array}{c}\text { Line } \\
\text { capacity } \\
\text { per hour at } \\
100 \%\end{array}$} & \multirow[b]{2}{*}{$\begin{array}{c}\text { Line } \\
\text { capacity per } \\
\text { hour at } 90 \%\end{array}$} & \multirow[b]{2}{*}{$\begin{array}{c}\text { Actual line } \\
\text { output per } \\
\text { hour }\end{array}$} & \multirow[b]{2}{*}{ Remarks } \\
\hline S. No & Name & & & & & & & & \\
\hline & & & & & & $100 \%$ & $90 \%$ & & \\
\hline 1 & Moina & $\begin{array}{l}\text { Back and front } \\
\text { match }\end{array}$ & 1 & 0.33 & 180 & 180 & 162 & 140 & \\
\hline 2 & Zorina & Attach shoulder & 1 & 0.34 & 176 & 176 & 158 & 145 & \\
\hline 3 & Sokina & $\begin{array}{l}\text { Tack and fold neck } \\
\text { rib }\end{array}$ & 1 & 0.33 & 184 & 184 & 165 & 142 & \\
\hline 4 & Aduri & $\begin{array}{l}\text { Attach neck rib to } \\
\text { neck }\end{array}$ & 1 & 0.32 & 190 & 190 & 171 & 145 & \\
\hline 5 & Karima & Back neck piping & 1 & 0.33 & 182 & 182 & 164 & 140 & \\
\hline 6 & Rekha & $\begin{array}{l}\text { Back neck piping } \\
\text { thread cut }\end{array}$ & 1 & 0.25 & 244 & 244 & 220 & 142 & Bottle neck \\
\hline 7 & Laizu & $\begin{array}{l}\text { Back piping nose } \\
\text { tack }\end{array}$ & 1 & 0.31 & 195 & 195 & 175 & 140 & \\
\hline 8 & Zosna & $\mathrm{T} / \mathrm{S}$ back neck & 1 & 0.34 & 174 & 174 & 157 & 142 & \\
\hline 9 & Hosna & $\mathrm{T} / \mathrm{S}$ front neck & 1 & 0.33 & 180 & 180 & 162 & 140 & \\
\hline 10 & Rabeya & Hem sleeve & 2 & 0.33 & 368 & 368 & 331 & 145 & Bottle neck \\
\hline 11 & Halima & Match sleeve to body & 1 & 0.32 & 188 & 188 & 169 & 138 & \\
\hline 12 & Beauty & $\begin{array}{l}\text { Attach sleeve to } \\
\text { body }\end{array}$ & 2 & 0.41 & 292 & 292 & 263 & 140 & Bottle neck \\
\hline 13 & Rehena & Close side seam & 2 & 0.65 & 184 & 184 & 165 & 140 & \\
\hline 14 & Kolpona & $\begin{array}{l}\text { Sleeve opening } \\
\text { horizontal and } \\
\text { verical tack }\end{array}$ & 2 & 0.69 & 174 & 174 & 157 & 138 & \\
\hline 15 & Sathi & Fold body for hem & 1 & 0.34 & 176 & 176 & 158 & 140 & \\
\hline 16 & Bithi & Sweing body hem & 1 & 0.32 & 190 & 190 & 171 & 138 & \\
\hline 17 & Rohima & $\begin{array}{l}\text { Sweing body hem } \\
\text { thread cut }\end{array}$ & 1 & 0.27 & 224 & 224 & 202 & 138 & Bottle neck \\
\hline 18 & Sumi & Final thread cut & 2 & 0.57 & 210 & 210 & 189 & 138 & \\
\hline
\end{tabular}


Efficiency Calculation before Work-Sharing:

Given that,

Manpower $=23$

Working hour $=10$

Actual Output $=1380 / 10$ hour

SMV $=5.50$

Efficiency $=($ Line Output X SMV $) /($ Manpower X Working Hour $) X 100 \%$

$$
\begin{aligned}
& =(1380 \times 5.5) /(23 \times 10 \times 60) \times 100 \% \\
& =55 \%
\end{aligned}
$$

\section{Experimental data (After work-sharing)}

From the graph, it has been seen that the capacity fluctuation was removed by removing the bottleneck (Figure 2). To do that, four manpower was reduced and some of the work was shared with other workers as shown in the Table 2.

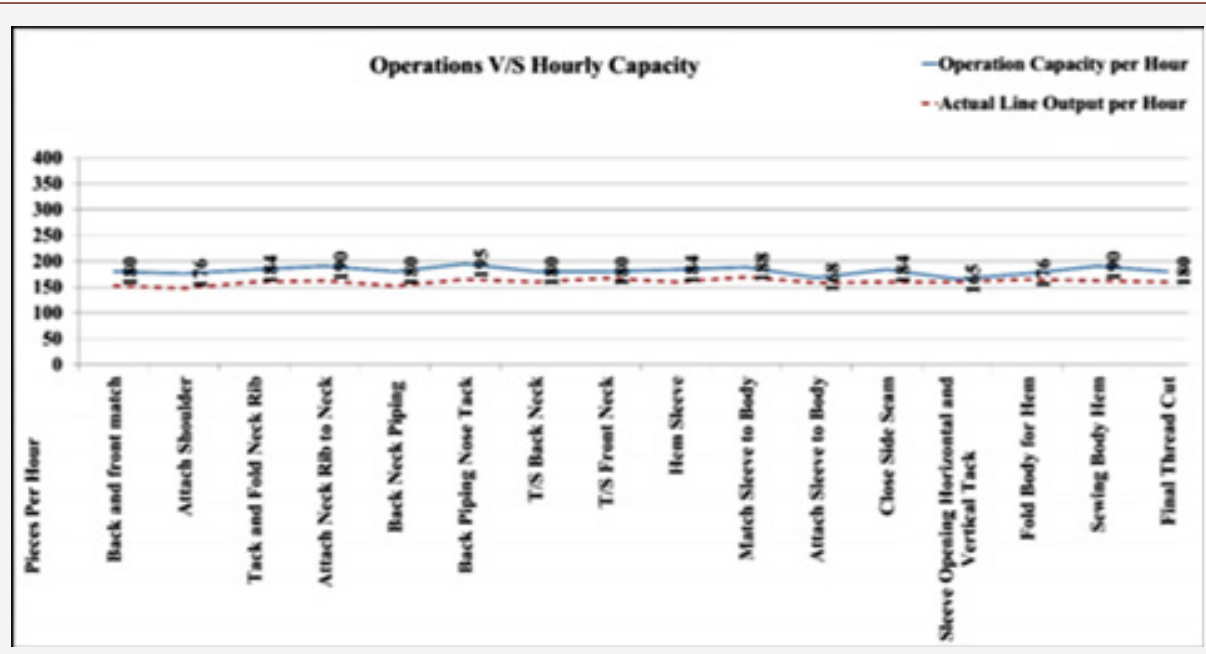

Figure 2: Operations vs. hourly capacity.

\begin{tabular}{|c|c|c|c|c|c|c|c|c|c|}
\hline \multicolumn{10}{|c|}{ Mahadi Fashion (Pvt) Ltd } \\
\hline \multicolumn{10}{|c|}{ Capacity study based pitch diagram } \\
\hline \multicolumn{2}{|c|}{ Buyer: Sisal } & \multirow{2}{*}{$\begin{array}{l}\text { Sty \# } 6704 \\
\text { Operations }\end{array}$} & \multirow{2}{*}{$\begin{array}{c}\text { SMV } \\
\begin{array}{c}\text { No of } \\
\text { operators }\end{array}\end{array}$} & \multirow{2}{*}{$\begin{array}{c}5.5 \\
\text { Cycle time with } \\
\text { allowance (In } \\
\text { minutes) }\end{array}$} & \multirow[b]{2}{*}{$\begin{array}{c}\text { Operation } \\
\text { capacity per } \\
\text { hour }\end{array}$} & \multirow[b]{2}{*}{$\begin{array}{c}\text { Line capacity } \\
\text { per hour at } \\
100 \%\end{array}$} & \multirow[b]{2}{*}{$\begin{array}{c}\text { Line } \\
\text { capacity per } \\
\text { hour at } 90 \%\end{array}$} & \multirow[b]{2}{*}{$\begin{array}{l}\text { Actual line } \\
\text { output per } \\
\text { hour }\end{array}$} & \multirow[b]{2}{*}{ Remarks } \\
\hline S. No & Name & & & & & & & & \\
\hline & & & & & & $100 \%$ & $90 \%$ & & \\
\hline 1 & Moina & $\begin{array}{l}\text { Back and front } \\
\text { match }\end{array}$ & 1 & 0.33 & 180 & 180 & 162 & 152 & \\
\hline 2 & Zorina & Attach shoulder & 1 & 0.34 & 176 & 176 & 158 & 148 & \\
\hline 3 & Sokina & $\begin{array}{l}\text { Tack and fold neck } \\
\text { rib }\end{array}$ & 1 & 0.33 & 184 & 184 & 165 & 160 & \\
\hline 4 & Aduri & $\begin{array}{c}\text { Attach neck rib to } \\
\text { neck }\end{array}$ & 1 & 0.32 & 190 & 190 & 171 & 162 & \\
\hline 5 & Karima & Back neck piping & 1 & 0.35 & 180 & 180 & 162 & 152 & $\begin{array}{c}\text { Bottle neck } \\
\text { piping thread } \\
\text { cute was done } \\
\text { by Karima }\end{array}$ \\
\hline 6 & Laizu & $\begin{array}{l}\text { Back piping nose } \\
\text { tack }\end{array}$ & 1 & 0.31 & 195 & 195 & 175 & 165 & \\
\hline 7 & Zosna & $\mathrm{T} / \mathrm{S}$ back neck & 1 & 0.34 & 180 & 180 & 162 & 160 & \\
\hline 8 & Hosna & $\mathrm{T} / \mathrm{S}$ front neck & 1 & 0.33 & 180 & 180 & 162 & 167 & \\
\hline 9 & Rabeya & Hem sleeve & 1 & 0.33 & 184 & 184 & 165 & 160 & $\begin{array}{l}\text { Reduce one } \\
\text { worker }\end{array}$ \\
\hline 10 & Halima & $\begin{array}{l}\text { Match sleeve to } \\
\text { body }\end{array}$ & 1 & 0.32 & 188 & 188 & 169 & 169 & \\
\hline 11 & Beauty & $\begin{array}{l}\text { Attach sleeve to } \\
\text { body }\end{array}$ & 1 & 0.39 & 168 & 168 & 162 & 158 & $\begin{array}{l}\text { Reduce one } \\
\text { worker }\end{array}$ \\
\hline
\end{tabular}

Table 2: Capacity study after work-sharing. 


\begin{tabular}{|c|c|c|c|c|c|c|c|c|c|}
\hline 12 & Rehena & Close side seam & 2 & 0.65 & 184 & 184 & 165 & 160 & \\
\hline 13 & Kolpona & $\begin{array}{c}\text { Sleeve opening } \\
\text { horizontal and } \\
\text { verical tack }\end{array}$ & 2 & 0.73 & 165 & 165 & 149 & 160 & \\
\hline 14 & Sathi & Fold body for hem & 1 & 0.34 & 176 & 176 & 158 & 165 & \\
\hline 15 & Bithi & Sweing body hem & 1 & 0.32 & 190 & 190 & 171 & 162 & \\
\hline 16 & Sumi & Final thread cut & 2 & 0.6 & 180 & 180 & 162 & 160 & $\begin{array}{l}\text { Sewing body } \\
\text { hem thread cut } \\
\text { was done by } \\
\text { Sumi }\end{array}$ \\
\hline
\end{tabular}

Efficiency Calculation after Work-Sharing:

Given that,

Manpower $=19$

Working hour $=10$

Actual line output $=1600 / 10$ hour

$\mathrm{SMV}=5.50$

Efficiency $=($ Line output X SMV $) /($ Manpower $X$ Working Hour $) X 100 \%$

$$
\begin{aligned}
& =(1600 X 5.5) /(19 X 10 X 60) X 100 \% \\
& =77 \%
\end{aligned}
$$

\section{Reduction of bottleneck by lean application}

Here, used time study to balance these sewing lines which is a part of work study. It implements the use of SMV calculation to identify the points where production has gone below the standard

Table 3: Traditional operational break-down (Style Name : Polo Shirt). level and the places where the production is above the standard. Then it is balanced to remove bottle neck in order to increase productivity. This system was effective and helpful. Considerable improvement observed by using time study as a line balancing technique changing form traditional layout to balanced layout model. The exchanges of work between the operator \& helper caused a significant change in line results of reducing wastage of time, minimum no. of worker and which caused high productivity in the manufacturing process. This balancing process also leads to increased output per day, labor productivity, machine productivity and overall line efficiency. The overall results relay on maximum profit of the company with effective use of its available resources. Our efforts and analysis say it is an effective method that helps to increase productivity. It is easy and can be applied in a simple way. But to sustain in the competitive market we need to gear more productivity. Here lean can help us to get the right results as it has

\begin{tabular}{|c|c|c|c|c|c|c|c|}
\hline S. No & Operations Name & Machine & No of Workers & Actual Time (Sec) & Allowance & Standard Time (Sec) & Capacity \\
\hline 1 & Placket Mark & Table & 1 & 12 & 2.4 & 14.4 & 257 \\
\hline 2 & $\begin{array}{l}\text { Placket role + Body } \\
\text { match }\end{array}$ & Over lock m/c & 1 & 15 & 3 & 18 & 200 \\
\hline 3 & Attached Placket & Plain m/c & 1 & 17 & 0.3 & 20.4 & 180 \\
\hline 4 & Placket fold tuck & Plain m/c & 1 & 12.6 & 1.6 & 8.4 & 450 \\
\hline 5 & Front back machine & Table & 1 & 12 & 2.4 & 14.4 & 257 \\
\hline 6 & Shoulder join + cut & Table & 1 & 10 & 2 & 12 & 300 \\
\hline 7 & Care label attach & Plain m/c & 1 & 21 & 4.2 & 25.2 & 144 \\
\hline 8 & Collar marking & Table & 1 & 11 & 2.2 & 13.2 & 277 \\
\hline 9 & Collar join & Plain $\mathrm{m} / \mathrm{c}$ & 1 & 28 & 5.6 & 33.6 & 106 \\
\hline 10 & Sleeve match & Table & 1 & 8 & 1.6 & 9.6 & 360 \\
\hline 11 & Sleeve join with body & Over lock m/c & 1 & 8 & 1.6 & 9.6 & 360 \\
\hline 12 & Sleeve join & Over lock m/c & 1 & 20 & 3.6 & 26.6 & 160 \\
\hline 13 & Collar binding & Plain m/c & 1 & 10 & 2 & 12 & 300 \\
\hline 14 & Binding cut + over turn & Table & 1 & 10 & 2.2 & 12.4 & 270 \\
\hline 15 & Collar top stitch & Plain m/c & 1 & 11 & 2.2 & 13.2 & 277 \\
\hline 16 & Placket close (r) & Plain m/c & 1 & 11 & 2.2 & 13.2 & 277 \\
\hline 17 & Placket close (l) & Plain $\mathrm{m} / \mathrm{c}$ & 1 & 12 & 2.4 & 14.4 & 257 \\
\hline 18 & Make Placket Box & Plain m/c & 1 & 42 & 8.4 & 50.4 & 71 \\
\hline 19 & Make Placket box & Plain m/c & 1 & 19 & 3.8 & 22.8 & 157 \\
\hline 20 & Arm hole join & Flat lock m/c & 1 & 14 & 2.2 & 16.2 & 198 \\
\hline 21 & Side seam & Over lock m/c & 1 & 28 & 5.6 & 33.6 & 106 \\
\hline
\end{tabular}
some more potential tools and systems (Table 3 \& Table 4). 


\begin{tabular}{|c|c|c|c|c|c|c|c|}
\hline 22 & Side seam & Over lock m/c & 1 & 21 & 4.2 & 25.2 & 144 \\
\hline 23 & Placket tick & Plain m/c & 1 & 14 & 3.6 & 22.7 & 167 \\
\hline 24 & Body hem & Flat lock m/c & 1 & 9 & 1.8 & 10.8 & 327 \\
\hline 25 & Sleeve hem & Flat lock m/c & 1 & 12 & 2.4 & 14.4 & 257 \\
\hline 26 & Button holing & Button holing m/c & 1 & 7 & 1.4 & 8.4 & 406 \\
\hline 27 & Button attaching & Button attaching m/c & 1 & 12 & 2.4 & 14.4 & 406 \\
\hline 28 & Thread cutting & Cutter & 2 & 45 & 9 & 54 & 67 \\
\hline & & & Total=29 & & & Total=523.5 \\
\hline
\end{tabular}

Table 4: Lean line operational break-down (Style Name : Polo Shirt).

\begin{tabular}{|c|c|c|c|c|c|c|c|}
\hline SL. No. & Operations Name & Machine & No of Workers & Actual time (Sec) & Allowance & Standard Time (Sec) & Capacity \\
\hline 1 & Placket Mark & Table & 1 & 11 & 2.2 & 13.2 & 277 \\
\hline 2 & Placket role + Body match & Over lock m/c & 1 & 12 & 2.4 & 14.4 & 258 \\
\hline 3 & Attached Placket & Plain m/c & 1 & 15 & 3 & 18 & 200 \\
\hline 4 & Placket fold tuck & Plain m/c & 1 & 9 & 1.8 & 10.8 & 333 \\
\hline 5 & Front back machine & Table & 1 & 9 & 1.8 & 10.8 & 333 \\
\hline 6 & Shoulder join + cut & Table & 1 & 16 & 3.32 & 19.2 & 187 \\
\hline 7 & Care label attach & Plain m/c & 1 & 20 & 4 & 24 & 150 \\
\hline 8 & Collar marking & Table & 1 & 17 & 3.4 & 20.4 & 177 \\
\hline 9 & Collar join & Plain m/c & 1 & 19 & 3.8 & 22.8 & 157 \\
\hline 10 & Sleeve match & Table & 1 & 4 & 0.8 & 4.8 & 750 \\
\hline 11 & Sleeve join with body & Over lock m/c & 1 & 35 & 7 & 42 & 86 \\
\hline 12 & Sleeve join & Over lock m/c & 1 & 23 & 4.6 & 27.6 & 130 \\
\hline 13 & Collar binding & Plain m/c & 1 & 15 & 3 & 18 & 200 \\
\hline 14 & Binding cut + over turn & Table & 1 & 12 & 2.4 & 14.4 & 250 \\
\hline 15 & Collar top stitch & Plain m/c & 1 & 14 & 2.8 & 16.8 & 214 \\
\hline 16 & Placket close (r) & Plain m/c & 1 & 16 & 3.2 & 19.2 & 188 \\
\hline 17 & Placket close (l) & Plain m/c & 1 & 15 & 3 & 18 & 200 \\
\hline 18 & Make Placket Box & Plain m/c & 1 & 42 & 8.4 & 50.4 & 71 \\
\hline 19 & Make Placket box & Plain m/c & 1 & 29 & 5.8 & 34.8 & 103 \\
\hline 20 & Arm hole join & Flat lock m/c & 1 & 16 & 3.2 & 19.2 & 188 \\
\hline 21 & Side seam & Over lock m/c & 1 & 40 & 8 & 48 & 75 \\
\hline 22 & Side seam & Over lock m/c & 1 & 33 & 6.6 & 39.6 & 91 \\
\hline 23 & Placket tick & Plain m/c & 1 & 19 & 3.8 & 22.8 & 157 \\
\hline 24 & Body hem & Flat lock m/c & 1 & 13 & 2.6 & 15.6 & 230 \\
\hline 25 & Sleeve hem & Flat lock m/c & 1 & 19 & 3.8 & 22.8 & 157 \\
\hline 26 & Button holing & Button holing m/c & 1 & 18 & 3.6 & 21.6 & 167 \\
\hline 27 & Button attaching & Button attaching $\mathrm{m} / \mathrm{c}$ & 1 & 5 & 1 & 6 & 600 \\
\hline \multirow[t]{2}{*}{28} & Thread cutting & Cutter & 2 & 45 & 9 & 54 & 67 \\
\hline & & & Total $=29$ & & & Total $=649.2$ & \\
\hline
\end{tabular}

\section{Productivity $=$ Output $/$ Input X100\%}

$$
\begin{aligned}
& =110 / 160 \times 100 \% \\
& =68.75 \%
\end{aligned}
$$$$
\operatorname{SMV}=511.6 / 60
$$$$
=8.53
$$

Standard SMV $=7.78$

SMV increased $=(8.53-7.78) / 7.78 \times 100 \%$

\section{$=9.6 \%$}

Efficiency \% of Line $=($ Total production X SMV X100) $/($ No. of Working Hour X No. of Workersx60)

$=(110 \times 8.53 \times 100) /(37 \times 1 \times 60)$

$=42.27 \%$

SMV target fulfilment $=(160-110) / 160 \mathrm{X} 100 \%$

$$
\begin{aligned}
& =100 \%-31.25 \% \\
& =68.75 \%
\end{aligned}
$$

Basic peace time $(\mathrm{BPT})=$ Total Time $/$ Total Manpower 


$$
\begin{aligned}
& =511.6 / 37 \\
& =13.827 \mathrm{sec}
\end{aligned}
$$

Capacity $/$ Hour $=3600 / 13.827$

$$
=260 \mathrm{pcs}
$$

Productivity $=$ Output $/$ Input X100\%

$$
\begin{aligned}
& =140 / 160 \times 100 \% \\
& =87.5 \%
\end{aligned}
$$

$\operatorname{SMV}=649.2 / 60$

$$
=10.82
$$

Standard SMV=12.94

SMV Decreased $=(12.94-10.82) / 10.82 \times 100 \%$

$$
=19.6 \%
$$

Efficiency $\%$ of Line $=($ Total production $X$ SMV X100)/(Number of OP X Working Hours $X 60)$
$=(140 \times 10.82 \times 100) /(29 \times 1 \times 60)$

$=87.05 \%$

SMV Target Fulfilment $=(140-160) / 160 \times 100 \%$

$$
\begin{aligned}
& =100 \%-12.5 \% \\
& =87.5 \%
\end{aligned}
$$

Basic peace time $(\mathrm{BPT})=$ Total Time $/$ Total Manpower

$$
\begin{aligned}
& =649.2 / 29 \\
& =22.38 \mathrm{sec}
\end{aligned}
$$

Capacity $/$ Hour $=3600 /$ BPT

$$
\begin{aligned}
& =3600 / 22.38 \\
& =160 \mathrm{pcs}
\end{aligned}
$$

\section{Transportation analysis traditional line vs. lean line}

(Table 5 and Figure 3)

Table 5: Transportation analysis traditional line vs. lean line.

\begin{tabular}{|c|c|c|c|c|c|c|}
\hline KPI & Unit of measure & Traditional line & Avg & Lean line & Avg & Improvement \\
\hline \multirow{5}{*}{ Transportation } & \multirow{3}{*}{ Feet } & 208 & & 99 & & \\
& & 209 & & 100 & \\
& & 208 & 209 & 99 & $52.63 \%$ \\
& & 211 & & 103 & \\
\end{tabular}

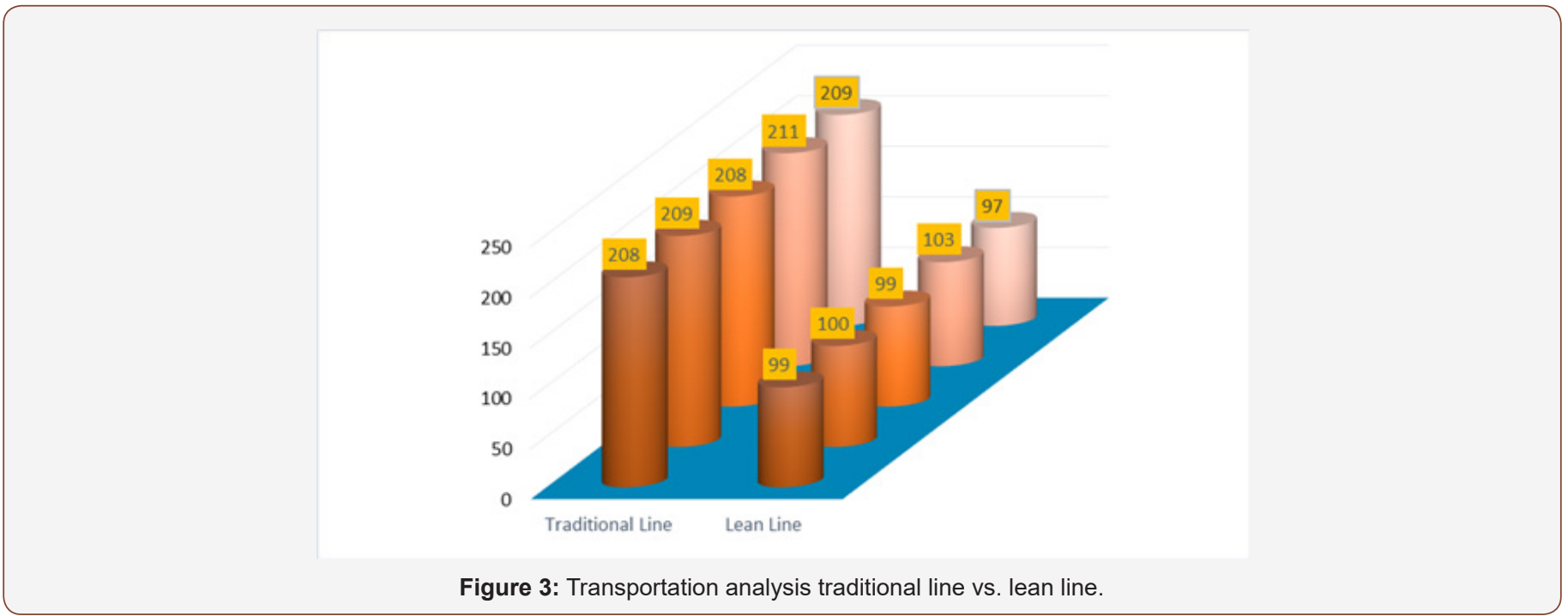

\section{Inventory analysis traditional line vs. lean line}

Lean manufacturing emphasizes the importance of reducing inventory, since it is considered to hide productivity problems

Table 6: Inventory analysis traditional line vs. lean line.

\begin{tabular}{|c|c|c|c|c|c|c|}
\hline KPI & Unit of measure & Traditional line & Avg & Lean line & Avg & Improvement \\
\hline \multirow{3}{*}{ Inventory/WIP } & \multirow{3}{*}{ Quantity } & 796 & & 352 & & \\
& & 790 & & 351 & & \\
& & 791 & 791 & 350 & & \\
& & 790 & & 352 & & \\
\hline
\end{tabular}

caused by unwanted variation and complicated set up procedures (Table 6 \& Figure 4). 


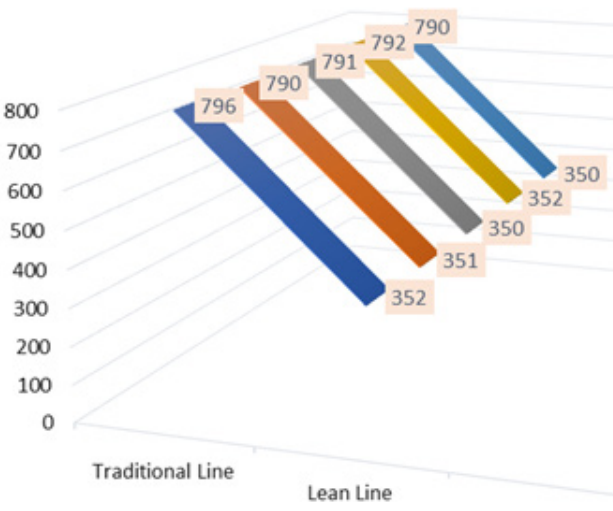

Figure 4: Inventory wip analysis traditional line vs. lean line.

\section{Space utilization analysis traditional line vs. lean line}

Table 7: Space utilization analysis traditional line vs. lean line.

\begin{tabular}{|c|c|c|c|c|c|c|}
\hline KPI & Unit of measure & Traditional line & Avg & Lean line & Avg & Improvement \\
\hline \multirow{5}{*}{ Space utilisation } & \multirow{3}{*}{ Minute } & 4.22 & & 3.62 & & \\
& & 4.5 & & 3.50 & & \\
& & 4.32 & 4.33 & 3.45 & 3.55 & \\
& & 4.2 & & 3.62 & & \\
\end{tabular}

Implementing lean in production system ensures maximum (Table 7 \& Figure 5). space utilization and thus reduces cycle time \& increases efficiency

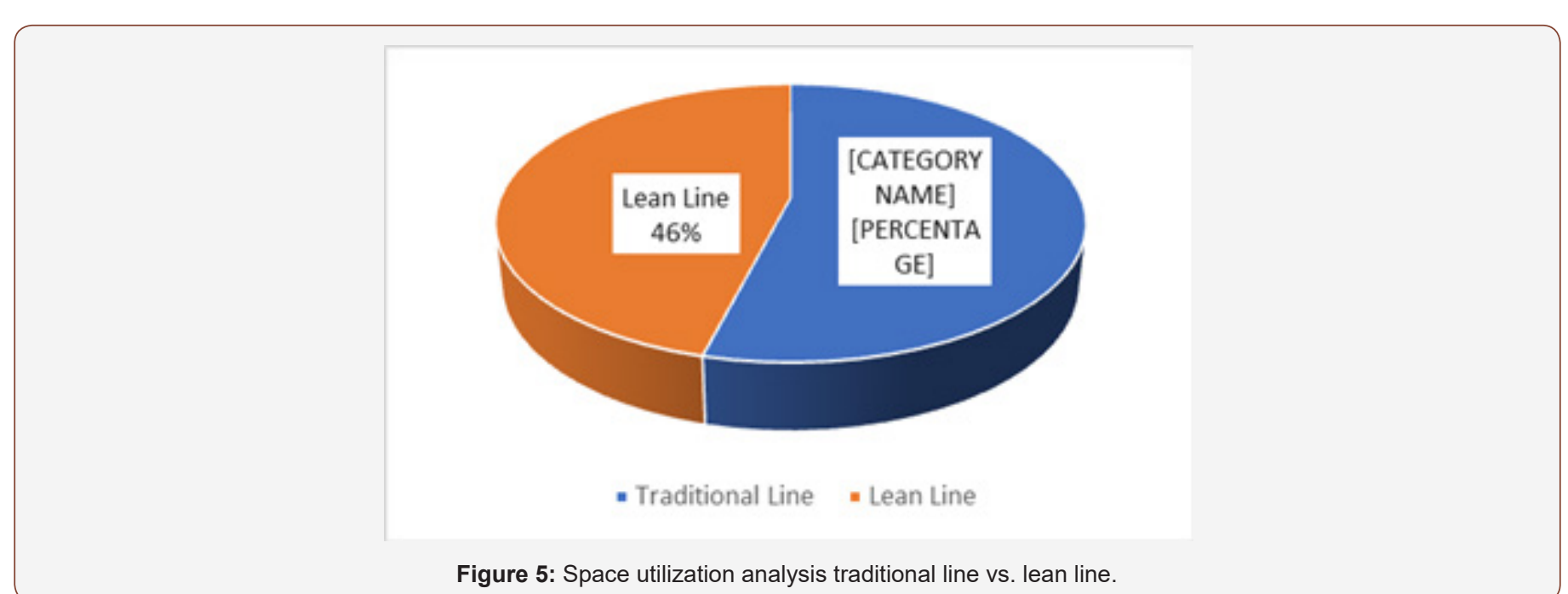

Work procedures at stations analysis traditional line vs.

\section{Lean line}

The work at the stations in the factory will to high extent be influenced by the implementation of lean. Some procedures will

Table 8: Work procedures at stations analysis traditional line vs. Lean line.

\begin{tabular}{|c|c|c|c|c|c|c|}
\hline KPI & Unit of measure & Traditional line & Avg & Lean line & Avg & Improvement \\
\hline \multirow{3}{*}{ Work station } & \multirow{3}{*}{ Quantity } & 22 & & 8 & & \\
& & 20 & & 6 & 7 & \\
& & 21 & 22 & 5 & 7 & \\
& & 24 & & 8 & & \\
\end{tabular}

probably have to be changed and improved, and it is therefore necessary to map the current work at the stations. Standardization, material handling, visualization and environment are investigated in this area (Table 8 \& Figure 6). 


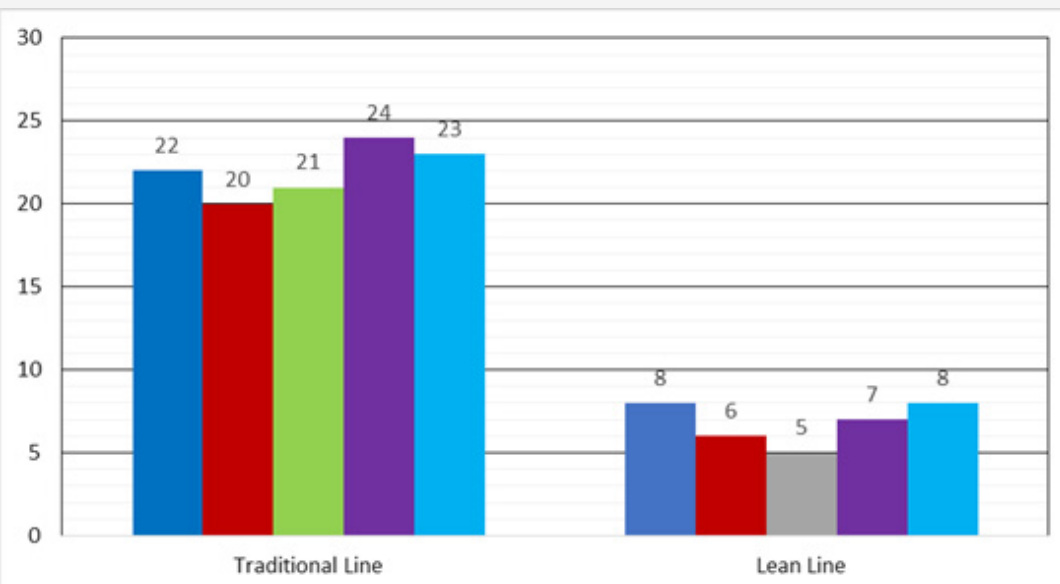

Figure 6: Work procedures at stations analysis traditional line vs. Lean line.

Different types of defects analysis traditional line $v s$.

\section{lean line}

Poor quality and the resulting defects are a major source of cost for many companies. This is also a cost that is often under reported as there are direct and indirect effects of defects. A defect is any error in a process that makes a product or service less valuable (Table 9 \& Figure 7).
Table 9: Different types of defects analysis traditional Line vs. Lean line.

\begin{tabular}{|c|c|c|}
\hline Defects & Traditional line & Lean line \\
\hline Seam puckering & 35 & 15 \\
\hline Slipped stitch & 25 & 7 \\
\hline Staggered stitch & 20 & 5 \\
\hline Thread breakage & 10 & 5 \\
\hline Variable stich density & 28 & 12 \\
\hline
\end{tabular}

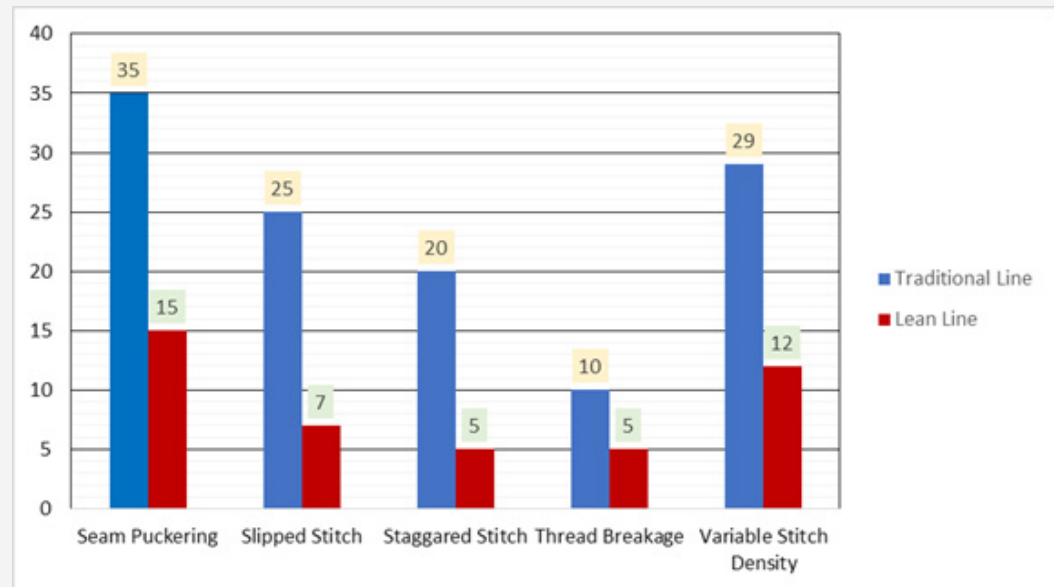

Figure 7: Different types of defects analysis traditional line vs. lean line.s

\section{Discussion of finding}

(Table 10 \&11)

Table 10: Comparing key productivity indicator for polo-shirt.

\begin{tabular}{|c|c|c|}
\hline Topic & Traditional line & Lean line \\
\hline Productivity & 68.75 & 87.5 \\
\hline Line efficiency & 42.27 & 87.05 \\
\hline SVM reduction & 9.6 & $19.60 \%$ \\
\hline SVM target fulfilment & 68.75 & 87.5 \\
\hline No of worker & 37 & 29 \\
\hline Bottle necks & 2 & Nil \\
\hline Capacity/hr utilisation & 110 & 140 \\
\hline
\end{tabular}

Table 11: Productivity analysis for polo-shirt.

\begin{tabular}{|c|c|c|c|c|}
\hline KPI & $\begin{array}{c}\text { Unit of } \\
\text { measure }\end{array}$ & $\begin{array}{c}\text { Traditional } \\
\text { line }\end{array}$ & Lean line & Improvement \\
\hline $\begin{array}{c}\text { Transport } \\
\text { analysis }\end{array}$ & Feet & 208 & 99 & $52.63 \%$ \\
\hline Inventory & Quantity & 796 & 352 & $55.62 \%$ \\
\hline $\begin{array}{c}\text { Space } \\
\text { utilisation }\end{array}$ & Min & 5.77 & 4.12 & $18.01 \%$ \\
\hline $\begin{array}{c}\text { Work } \\
\text { station }\end{array}$ & Quantity & 25 & 16 & $68.18 \%$ \\
\hline
\end{tabular}

\section{Conclusion}

Bottleneck reduction is an important issue in RMG sector. Because it is related with productivity improvement. The profit 
earning of apparel industry largely depends on productivity improvement. This study shows the way of improving the production efficiency by bottleneck reduction. Number of operators is reduced by considering process wise cycle time per head. A final layout for the finishing line is proposed. The proposed layout model has been followed the logic of modular system (worker works on more than two processes who is skilled on all processes and these combination of skilled workers finish their work in piece flow production) and traditional system (one worker works in one process and all the workers who may be skilled or not finish their work in bundle flow production) both together where only modular production system can be applicable with a series of skilled workers to achieve more productivity. This calculation could be justified by considering different line of the production floor. Result would have been more effective if the order considered is larger than the current order. Bottleneck reduction techniques are very important for reducing production time and improving quality.

\section{Acknowledgement}

None.

\section{Conflict of Interest}

No conflict of interest.

\section{References}

1. Haque MT, Hossain MR (2018) Bottleneck problem reduction of a garment manufacturing industry in Bangladesh by using line balancing technique. International Journal of Research in Advanced Engineering and Technology 4(2): 28-32.

2. Vilà $M$, Pereira J (2013) An enumeration procedure for the assembly line balancing problem based on branching by non-decreasing idle time, European Journal of Operational Research 229(1): 106-113.

3. Jayakumar AA, Krishnaraj C, Kumar AA (2017) Productivity improvement in stitching section of a garment manufacturing. International Journal of Innovative Research in Advanced Engineering 12(4):8-11.

4. Güner MG, Ünal C (2008) Line balancing in the apparel industry using simulation techniques, Fibres \& Textiles in Eastern Europe 16(2): 75-78.

5. Kishore VR, Rao YS (2018) A review of optimization techniques implementation in production industries. International Journal of Engineering and Techniques 4(1).

6. Marudhamuthu R, Krishnaswamy M, Pillai DM (2011) The Development and Implementation of Lean Manufacturing Techniques in Indian garment Industry,Jordan Journal of Mechanical and Industrial Engineering 5(6): 527-532.

7. Suman S (2018) Project report on achieving quick changeover through smed implementation at Laguna clothing Pvt. Ltd., Kanakapura.

8. Marudhamuthu R, Krishnaswamy M (2011) The development of green environment through lean implementation in a garment industry. ARPN Journal of Engineering and Applied Sciences 6(9): 104-111.

9. Bappy MM, Musa MA, Hossain MF (2019) Productivity improvement through line balancing-a case study in an apparel industry 7(2): 893902.

10. Alauddin Md (2018) Process improvement in sewing section of a garments factory - A case study. 